\title{
A new short version of the "Token Test" for Yawi native speakers
}

\author{
Phakkharawat Sittiprapaporn \\ Assistant Professor, Brain Science and Engineering Innovation Research Group, School of Anti-Aging and Regenerative \\ Medicine, Mae Fah Luang University, Bangkok, Thailand and Department of Anti-Aging and Regenerative Science, \\ School of Anti-Aging and Regenerative Medicine, Mae Fah Luang University, Bangkok, Thailand
}

Background: The Token Test as originally conceived by De Renzi and Vignol is a subtle test of receptive language functions. Although it has been employed in numerous clinical studies since 1962, no one has studied the linguistic properties of the commands in in Yawi-speaking aphasic patients. Aims and Objectives: The study aimed to describe the development of the Yawi Token Test (YWTT) and to investigate the test performances of the normal Yawi-speaking participants before applying with the Yawi-Speaking aphasic patients. Materials and Methods: An adaptation of the Yawi Token Test (YWTT) was administered to one-hundred normal Yawi-speaking participants, ranging in age from 18-45 years, with minimal educational level of Prathom 4 who were living in Pattani Province, South of Thailand. Results: Performance on Part I-V and overall performance were reported. Overall, participants in the trial version performed not significantly on the Yawi Token Test (YWTT) overall composite score compared to final version. The mean Yawi Token Test (YWTT) score of the trail and final versions were 59.40 (S.D. $=1.29$; range: $56-61$ ) and 60.44 (S.D. = 1.39; range: $56-61$ ), respectively. The mean Yawi Token Test (YWTT) score for overall (100 participants) was 60.42 (S.D. $=1.32$; range score: $56-61$ ). Comparing with the trial version, participants did obviously lower number of errors of all parts in the final version. Conclusion: Yawi Token Test (YWTT) was applicable to the differential diagnosis of the communicative abilities of Yawi-speaking aphasic patients. This test will be helpful for assessing auditory language comprehension Yawi-speaking aphasic patients.
http://nepjol.info/index.php/AJMS DOI: 10.3126/ajms.v10i5.25044 E-ISSN: 2091-0576 P-ISSN: 2467-9100

Key words: Token Test; Yawi Language; Aphasia

\section{INTRODUCTION}

To establish a patient understands speech is vital to many aspects of a neuropsychological investigation. Communication difficulties usually preclude the evaluation of speech comprehension as shown in a variety of neurological diseases. ${ }^{1}$ As auditory processing and comprehension are psycho-physiologically and linguistically complex enterprises, the importance of auditory processing and comprehension in aphasic individuals and the pervasiveness of auditory processing impairments in aphasia have been repeatedly demonstrated ${ }^{2-4}$ and described by some as the core disorder in aphasia. ${ }^{5}$ The array of tests and measures that have been developed to assess the multiple levels of the auditory system reflects its extreme complexities. ${ }^{1}$

The Token Test as originally conceived by De Renzi and Vignol $^{6}$ is a subtle test of receptive language functions. De Renzi and Vignolo's Token Test represents an interesting technique to evaluate the verbal comprehension of aphasic patients. ${ }^{6}$ Using basic concepts of information theory, De Renzi and Vignol ${ }^{6}$ constructed verbal commands of various difficulty, but with practically zero redundancy: lacking any contextual help, the aphasic patients perform strikingly worse than the non-aphasic, brain damaged. ${ }^{1-4}$ The discriminative power of De Renzi and Vignolo's Token Test between aphasic and non-aphasic patients was quite 
higher than that of the great majority of the clinical tests used to evaluate linguistic deficit in comprehension. In addition, the Token Test is a particularly sensitive tool to detect very mild receptive disorders in aphasia. ${ }^{1-4}$

The Token Test is composed of tokens: two forms (rectangle and circle), two dimensions (large and small), and five different colors. Several studies have been explored more systematically the clinical validity of the Token Test. ${ }^{7-11}$ As the Token Test is a sensitive test for diagnosing aphasia, ${ }^{7,812}$ up to the present, no comprehensive Token Test has been available for use with Yawi-speaking aphasic patients in both Thailand and neighboring countries including Malaysia. This paper describes the first comprehensive Token Test to be developed for use with Yawi-speaking aphasic patients. The motivation of this Yawi Token Test development was twofold: (a) to find a means for assessing milder forms of receptive dysfunction which were difficult if not impossible to reveal by usual clinical methods in Yawi-speaking aphasic patients, and (b) to make the assessment purely linguistic in nature, i.e., uncontaminated by other intellectual functions, particularly attention span and the cognitive processing of a task in Yawi-speaking aphasic patients.

\section{MATERIALS AND METHODS}

\section{Participants}

One hundred normal Yawi-speaking participants from 18 years to 45 years from Pattani province, South of Thailand, were participated in this study. There were 75 females and 25 males in the sample. Their mean age was $19.30(\mathrm{SD}=5.77)$ years and their mean level of education was $13.27(\mathrm{SD}=1.0)$ years. All of the participants were dextral as assessed by the Edinburgh Handedness Inventory. ${ }^{13}$ No participant with clinically deviant speech was included. Criteria for inclusion were monolingual, Yawi-speaking language background, average intelligence, and native speaker of Yawi language. Exclusionary criteria included uncorrected visual or auditory deficits; motor disabilities that precluded manipulating tokens; prior diagnosis of mental retardation, language, and/or attention disorders; or recent diagnosis of clinical depression. In addition, all participants had normal or corrected to normal vision and were screened with a selfreport measure for previous audiological, psychiatric and/ or neurological disorders. The sole exclusion criterion was the presence of an existing problem (e.g., neurological) that could interfere with the cognitive functions required for the experiment. Informed consents were obtained and all of the participants were advised that the experimental session would be approximately twenty minutes in duration. This study had ethical committee approval.

\section{Yawi Token Test}

The short version of the Token Test ${ }^{14}$ was used as a standardized test, and it was administered in accordance with the instructions provided by Lezak. ${ }^{15}$ In this study the form of the Yawi Token Test was similar to the Token Test that described by Boller and Vignolo. The Yawi Token Test was designed to detect mild language comprehension disturbances for Yawi-speaking aphasic patients. The error Yawi Token Test score provides an indication of the presence or not of aphasia and a measure of overall severity of aphasic symptoms. Similar to the standardized test, the Yawi Token Test consisted of five subparts, each ten items long. The Yawi Token Test consisted of twenty tokens of two different shapes, two different sizes, and five different colors. The examinee manipulated tokens in response to oral instructions (Yawi Language) from the examiner who were native Yawi speaker. The Yawi Token Test was also divided into five parts, with each part presenting progressively longer and more complex spoken commands. Commands were syntactically similar in parts I-IV. Part IV introduced commands that demonstrated variations in linguistic concepts and structures. This short version consisted of 20 cardboard geometric shapes (tokens) and a list of 36 verbal commands separated into 6 parts. The tokens consisted of circles and squares in two sizes $(4 \mathrm{~cm}$ across and $8 \mathrm{~cm}$ across) and five colors including red, white, yellow, blue, and green, respectively. The verbal commands ranged from simple sentences (e.g., "Touch the circle") to more complex sentences (e.g., "Touch the black circle with the red square'). During the standardized test, the experimenter placed a fixed arrangement of 10 or 20 tokens (i.e., only the large tokens or both the large and the small tokens) on a table in front of the participant and read the commands aloud, slowly one at a time. The participant had been instructed to complete each verbal command and one point was awarded for a successful response. Items failed on the first command were repeated once. If the item was performed successfully the second time, then only the second response was scored. The initial error was assumed to result from non-specific factors, such as inattention. ${ }^{15}$ In this study, there were two versions of the Yawi Token Test; one was the "Trial" version and the other was "Final Concrete" version. Both versions of the Yawi Token Test were administered in each group of participant and the order was counterbalanced across participants. It was administered individually to each participant. Testing required approximately twenty minutes (Figure 1).

\section{Procedure}

All participants were examined with both versions of the Yawi Token Test; the interval between the two versions was 1 week. Half the participants (50 persons) received first the trial version and half received the final concrete version of the Yawi Token Test (50 persons).

\section{Data analysis}

Two-tailed t-tests were applied to assess for significant difference between standard scores for both trail and final 
concrete versions of the Yawi Token Test and the overall standard scores of the two groups at a $\mathrm{p}$ value of 0.05 .

\section{RESULTS}

Performance on Part I-V and overall performance are depicted in Table 1 and Figure 2. Overall, participants in the trial version performed not significantly $(t=0.14$; $\mathrm{df}=49$; $\mathrm{p}=0.89)$ on the Yawi Token Test overall composite score compared to final concrete version. The mean Yawi Token Test score of the trail and final concrete versions were 59.40 (S.D. $=1.29$; range: $56-61)$ and 60.44 (S.D. $=1.39$; range: $56-61$ ), respectively (Figure 3 ).

Additionally, the mean Yawi Token Test score for overall (100 participants) was 60.42 (S.D. $=1.32$; range score: 56 - 61) (Table 2 and Figure 4-5). Eight of the 100 participants scored 56-57 whereas 58-59 were shown

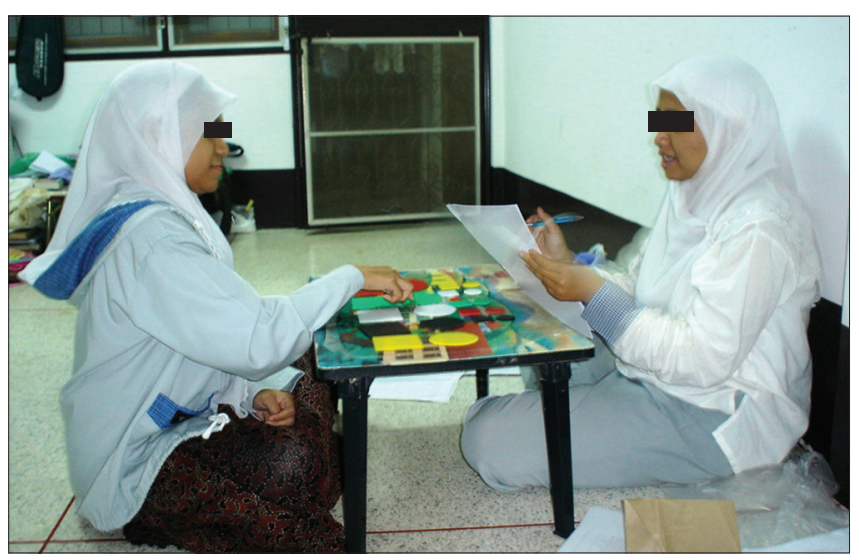

Figure 1: The examinee manipulated tokens in response to oral instructions (Yawi language) from the examiner who was native Yawi speaker

\begin{tabular}{lcc}
$\begin{array}{l}\text { Table 1: Performance on part I to V and overall } \\
\text { performance of Yawi Token Test }\end{array}$ \\
\hline Parts & Trial Version & Final Version \\
\hline Part I & 8.98 & 10 \\
Part II & 8.9 & 10 \\
Part III & 8.98 & 9.98 \\
Part IV & 8.92 & 9.98 \\
Part V & 19.62 & 20.48 \\
Overall & 59.4 & 60.44 \\
\hline
\end{tabular}

Table 2: Mean Yawi Token Test score for overall participants

\begin{tabular}{lcc}
\hline Token Test & Mean & Std. deviation \\
\hline Part I & 9.99 & 0.1 \\
Part II & 9.95 & 0.261 \\
Part III & 9.98 & 0.141 \\
Part IV & 9.95 & 0.219 \\
Part V & 20.55 & 1.123 \\
Overall & 60.42 & 1.319 \\
\hline
\end{tabular}

by only six participants. Most of the participants $(86 \%)$ reported 60-61.

The average number of errors for each part and the range of errors from all 100 participants is shown in Table 3. The average number of errors per command was calculated by obtaining the total number of errors for each part from trial and final concrete versions. Comparing with the trial version, participants did obviously lower number of errors of all parts in the final concrete version. The value of number of errors in both version are shown in Table 3 and Figure 6.

\section{DISCUSSION}

This study evaluated the efficiency of available screening tools to accurately identify potential language problems in Yawi aphasic patients. The study describes the usefulness of the Yawi Token Test for Yawi-speaking aphasic patients. The present study revealed subtle receptive language processing that potentially contributes to the language performance frequently reported among Yawi-speaking aphasic patients. Recognizing these facts, the present study is currently evaluating the efficiency of available screening tools to accurately identify potential language problems in Yawi-speaking aphasic patients. This report describes the usefulness of the Yawi Token Test for Yawi-speaking normal participants as an in-clinic language screen for Yawi-speaking aphasic patients.

Participants scored significantly higher overall in the Yawi Token Test. The behavioral analysis revealed that the participants performed equally well on the trial and the final concrete versions. The good performance in Part $\mathrm{V}$ of the Yawi Token Test is important. First, it might reflect a capacity in knowledge and comprehension of abstract linguistic concepts. Critical concepts and relationships featured in Part V of the Yawi Token Test are rich in abstract semantic categories including spatio-temporal and conditional concepts. ${ }^{15}$ Second, the good performance in Part V might reflect ability to hold information concurrently in short-term memory and process verbal directions varying in a number of linguistic elements. ${ }^{15}$

Compared to the normative data, ${ }^{14}$ the participants in this study scored in the average range on the trial and final concrete versions. Although these behavioral results are encouraging, care must be taken when making comparisons between standardized test and the Yawi Token Test. In order to adapt the Yawi Token Test for clinical use, more clinical trial with Yawi-speaking aphasic patients must be made. Ultimately, these recommended clinical trial might influence the cognitive processes required for successful performance on the Yawi Token Test. Success 


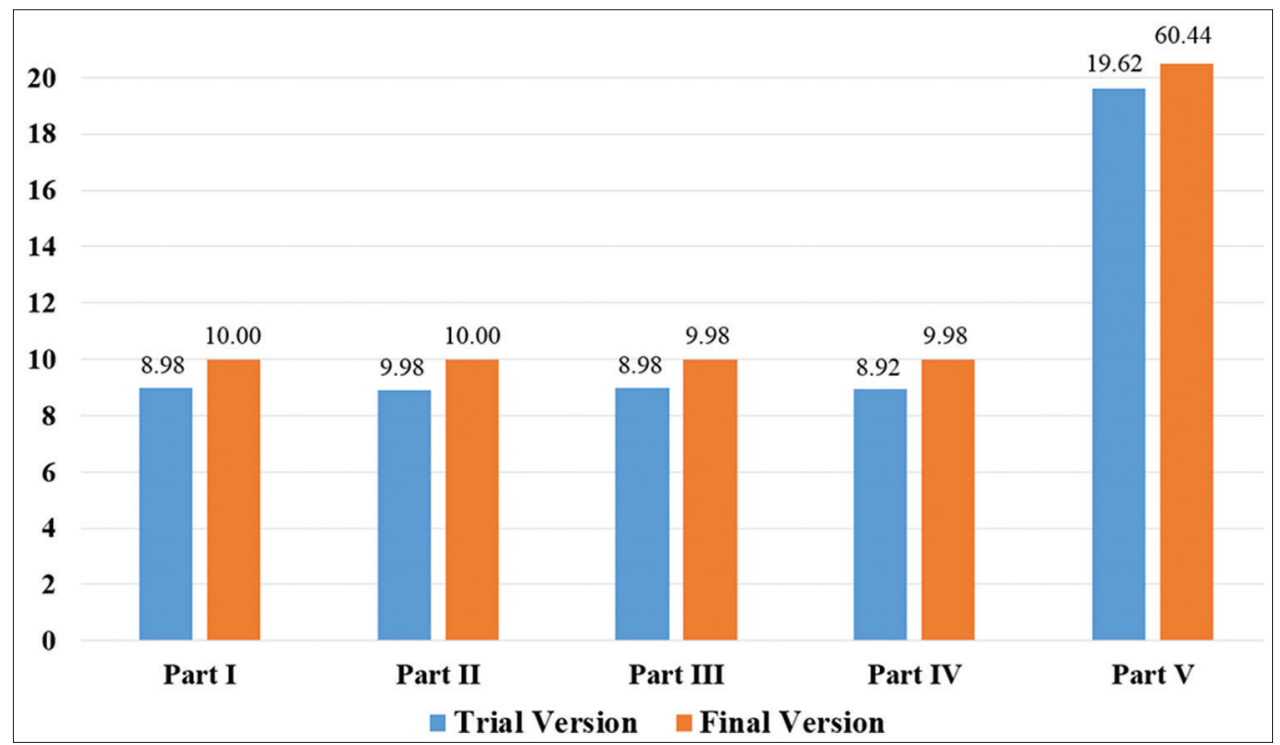

Figure 2: Performance on part I to $\mathrm{V}$ of Yawi Token Test

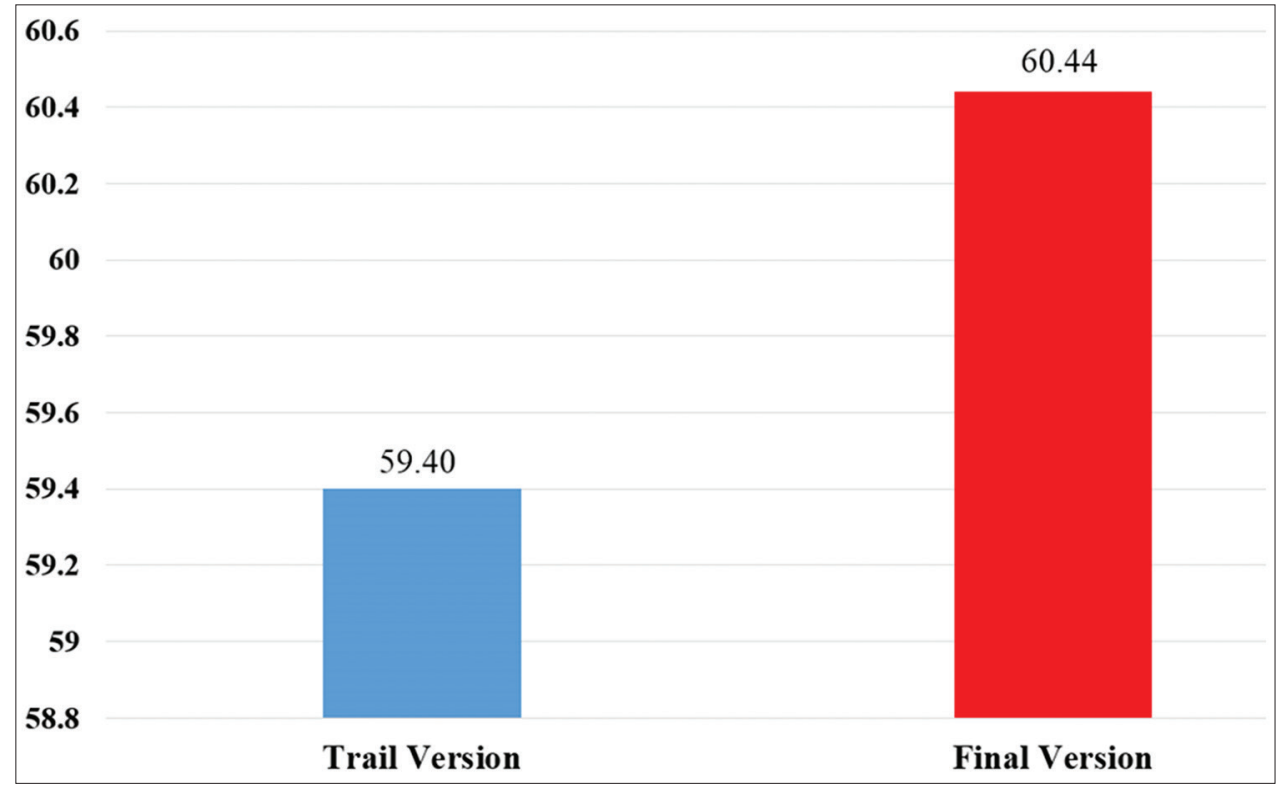

Figure 3: Overall performance of Yawi Token Test

Table 3: Average number and range of errors for each part of Yawi Token Test

\begin{tabular}{|c|c|c|c|c|c|}
\hline \multirow[t]{2}{*}{ Part } & \multicolumn{2}{|c|}{$\begin{array}{c}\text { Number of errors for each part for trial } \\
\text { version }\end{array}$} & \multicolumn{2}{|c|}{$\begin{array}{c}\text { Number of errors for each part for final } \\
\text { version }\end{array}$} & \multirow[t]{2}{*}{ Range of errors for each part } \\
\hline & Number of error & Percentage of error & Number of error & Percentage of error & \\
\hline I & 1 & 0.5 & 0 & 0.0 & $0-1$ \\
\hline II & 5 & 2.5 & 0 & 0.0 & $0-5$ \\
\hline III & 1 & 0.5 & 1 & 0.5 & $0-1$ \\
\hline IV & 4 & 2.0 & 1 & 0.5 & $1-4$ \\
\hline V & 26 & 13.0 & 19 & 9.5 & $19-26$ \\
\hline Over All & 30 & 15.0 & 28 & 14.0 & $28-30$ \\
\hline
\end{tabular}

on the Yawi Token Test for Yawi-speaking aphasic patients requires, at the very least, the ability to: (a) understand speech; (b) retain the information in working memory; and (c) generate a behavioral response in order to identify the correct token. Also, the design of the present study did not intend to distinguish between abstract language knowledge and working memory. Memory and language are inextricably related. ${ }^{16,17}$ Hence, if the memory capacity 


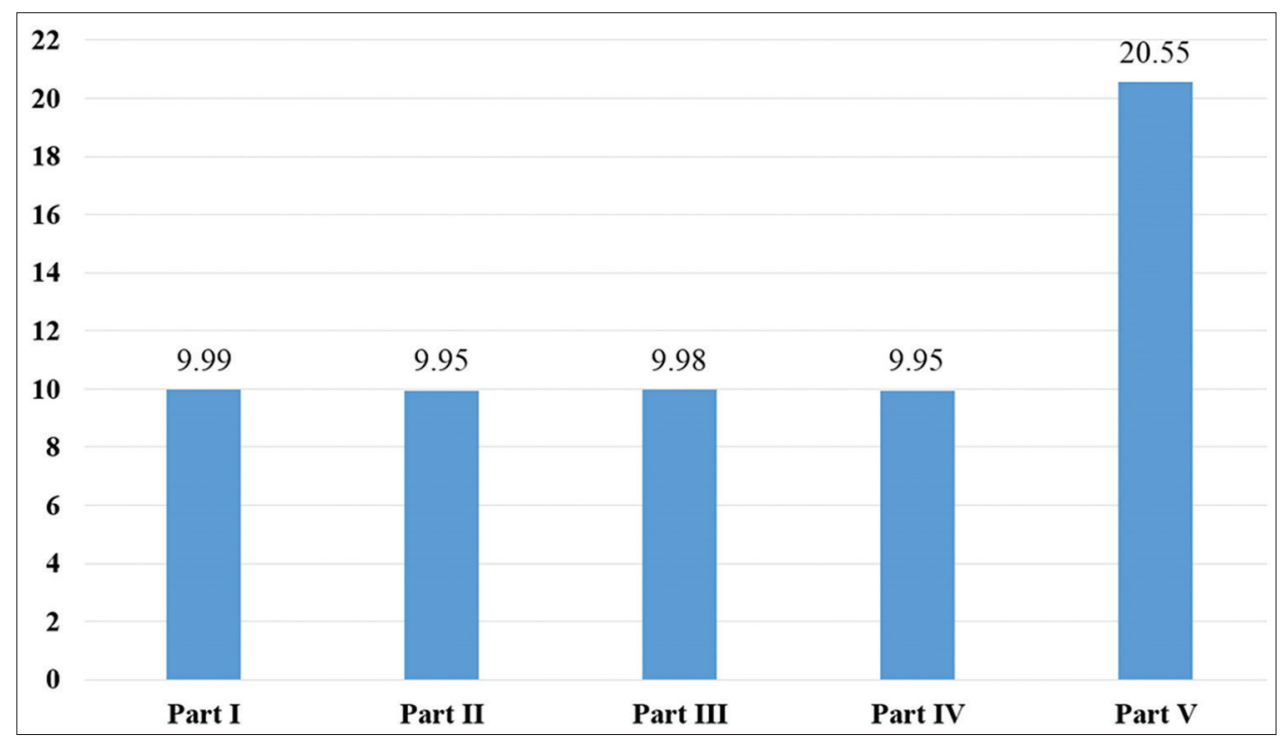

Figure 4: Mean Yawi Token Test score for each part by participants

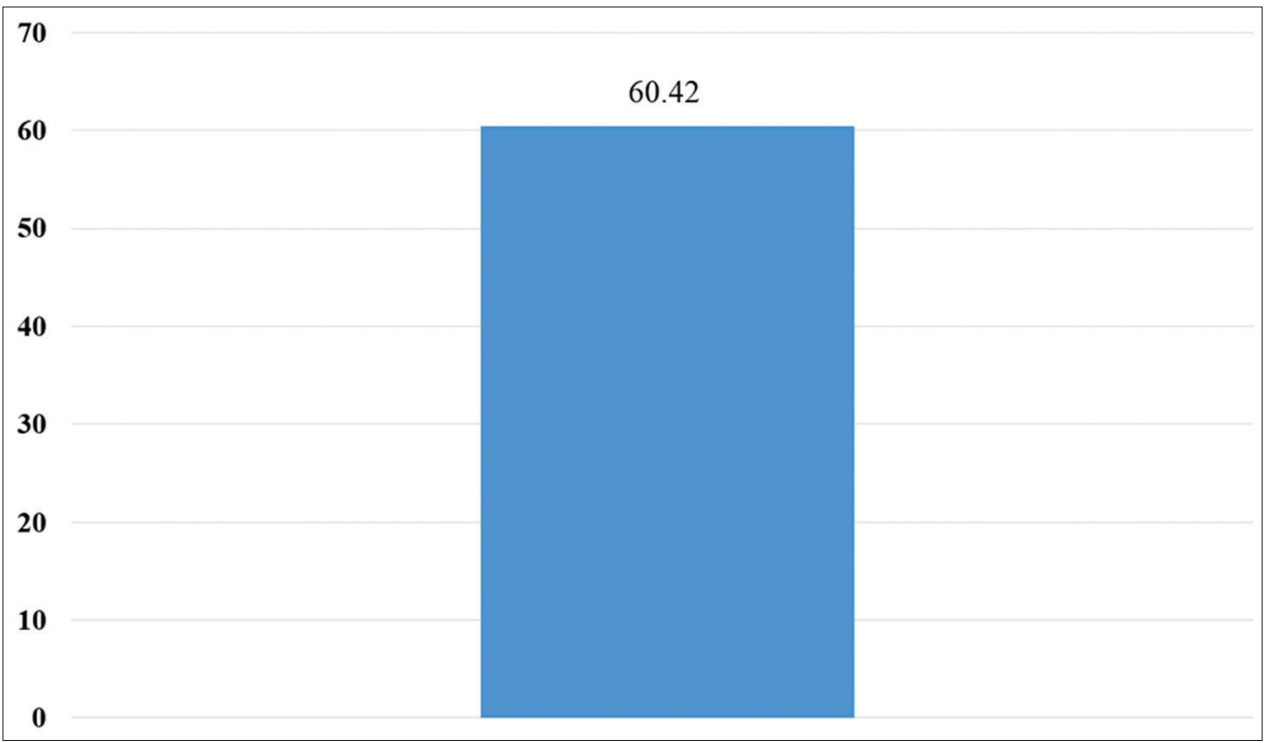

Figure 5: Mean Yawi Token Test score for overall participants

is not adequate for comprehending complex sentences, it should be considered a part of the language disorder, not apart from it. ${ }^{16}$

However, limitations to this study were also acknowledged. First, the current Yawi Token Test was tested for normal Yawi-speaking participants. This limitation dictates the need for replication in a larger sample of normal YawiSpeaking participants and Yawi-speaking aphasic patients. Second, while the Yawi Token Test is a receptive language measure, its primary receptive component is limited to knowledge of specific lexical semantics (color, shape, spatial, temporal, and conditional concepts), and to lesser extent knowledge of syntax. Nonetheless, the current Yawi Token Test is a potentially useful index of linguistic processing in Yawi-speaking aphasic patients. Language screening tools need to be highly sensitive to detect the varied and subtle language disabilities seen in Yawispeaking aphasic patient. From a practical and pragmatic viewpoint, the current Yawi Token Test served as a quick and functional in-clinic screen for selecting Yawi-speaking aphasic patients for more in-depth language assessment. Third, this study was concerned with only adults whereas several studies reported in the literature were concerned with children. For instance, a previous studies showed that the under 15-year-old group of children made significantly lower scores than the adult groups. ${ }^{8}$

The current Yawi Token Test is a measure of auditory comprehension designed to detect mild perceptive 


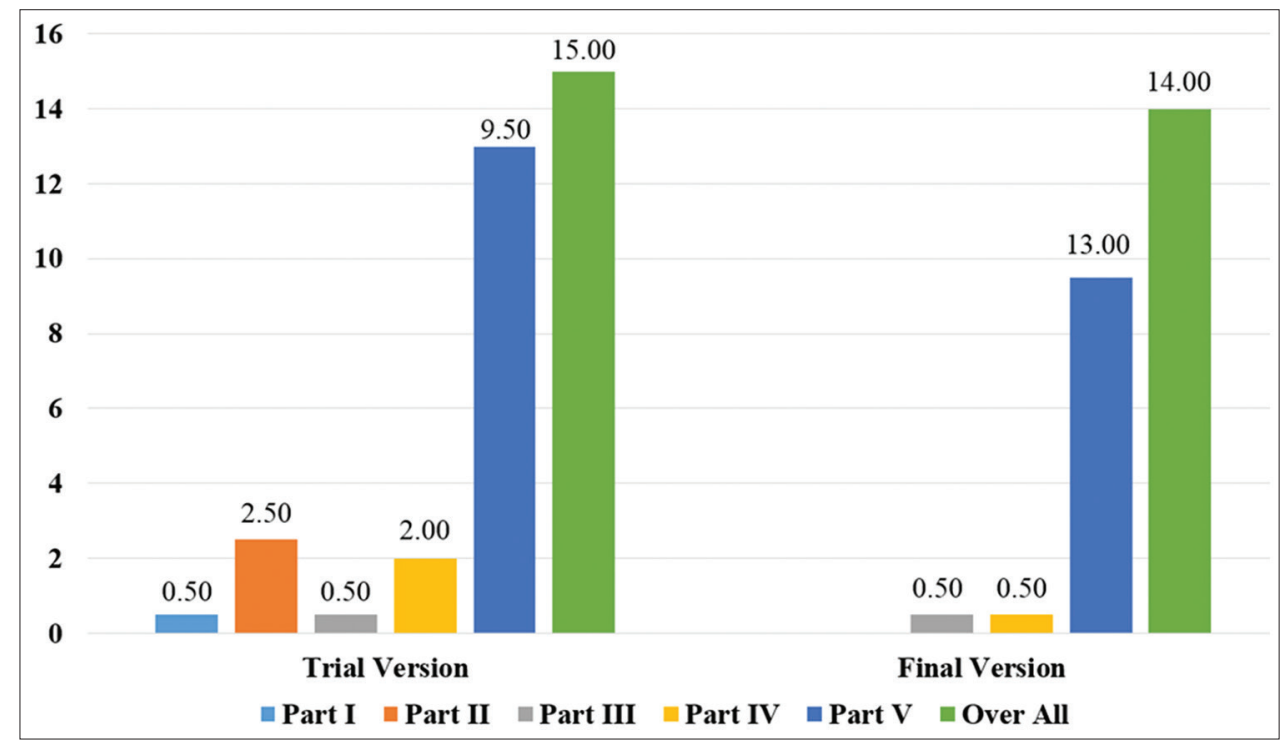

Figure 6: Percentage errors for each part of Yawi Token Test comparing between trial and final versions

disturbances in Yawi-speaking aphasic patients who demonstrate no apparent difficulty understanding auditory stimuli contained in traditional tests. Up to the present, no auditory comprehensive battery has been available for use with Yawi-speaking aphasic patients who live in region with shared border of Thailand and Malaysia. This study thus reported the first auditory comprehensive battery to be developed for use with Yawi-speaking aphasic patients. The result was similar to those of previous studies with the English and Thai version of the test in normal subjects. Consequently, the current Yawi Token Test will be helpful for detecting minimal auditory comprehension deficits in Yawi-speaking aphasic patients. Furthermore, it will be confined to evaluating auditory comprehension without any information concerning a patient's ability to read, speak, or write.

\section{CONCLUSION}

The present study developed the Yawi Token Test and investigated the test performances of the normal Yawispeaking participants before applying to the Yawi-speaking aphasic patients. As comparing with the trial version, participants did obviously lower number of errors of all parts in the final concrete version. The current Yawi Token Test is applicable to the differential diagnosis of the communicative abilities of the Yawi-speaking aphasic patients.

\section{ACKNOWLEDGEMENT}

We thank all of the participants who involved in this study as main data provider.

\section{REFERENCES}

1. D'Arcya RCN and Connolly, JF. An event-related brain potential study of receptive speech comprehension using a modified Token Test. Neuropsychologia 1999; 37:1477-1489.

2. Brookshire $\mathrm{RH}$. Differences in responding to auditory materials among aphasic patients. Acta Symbolica 1974; 1:1-18.

3. Square-Storer P, Darley F and Sommers R. Nonspeech and speech processing skills in patients with aphasia and apraxia of speech. Brain and Language, 1988; 33:65-85.

4. Murray L, Holland A and Beeson P. Auditory processing in individuals with mild aphasia: A study of resource allocation. Journal of Speech, Language, and Hearing Research 1997; 40: $792-808$

5. Schuell $\mathrm{H}$, Jenkins $\mathrm{J}$ and Jimenez-Pabon $\mathrm{E}$. Aphasia in adults. New York: Harper \& Row, 1964.

6. De Renzi E and Vignolo LA. The Token Test: a sensitive test to detect disturbances in aphasics. Brain 1962; 85:665-678.

7. Boller $F$ and Vignolo LA. Latent sensory aphasia in hemispheredamaged patients: an experimental study with the Token Test. Brain 1966; 89:815-831.

8. Orgass $B$ and Poeck K. Clinical validation of a new test for aphasia: an experimental study on the token test. Cortex 1966; 2:222-243.

9. Orgass B, Poeck K, Hartje W and Kerschensteiner M. Zum vorschlag einer kurzform des token test zur auslese von aphasikem. Nervenan 1973; 44:93-95.

10. Hartje W, Kerschensteiner M, Poeck K and Orgass B. A crossvalidation on the token test. Neuropsychologia 1973; 11: 119-121.

11. Von Dogen HR and Van Harskamp F. Token test. A preliminary evaluation of a method to detect aphasia. Psychiatr. Neural. Neurochir 1972; 75: 129-134.

12. De Renzi $\mathrm{E}$ and Vignolo LA. The token test: a sensitive test to detect receptive distorbancy in aphasics. Brain 1962; 85: 665-678.

13. Oldfield RC. The assessment and analysis of handedness: the Edinburgh inventory. Neuropsychologia 1971; 9:97-113.

14. De Renzi E and Faglioni P. Normative data and screening power of a shortened version of the Token Test. Cortex 1978; 14:41-49.

15. Lezak M. Neuropsychological assessment, $3^{\text {rd }}$ ed. New York: 
Oxford University Press, 1995

16. Vachha B and Adams RC. Application of the Token Test to Children with Myelomeningocele and Shunted Hydrocephalus.
Eur J Pediatr Surg 2002; 12:525-552.

17. Gathercole SE and Baddley AD. Working Momory and Language. Hillsdale, NJ: Erlbaum, 1993.

Author Contribution:

PS-Concept and design of the study, manuscript preparation, statistically analyzed and interpreted, critical revision of the manuscript.

Work attributed to:

Brain Science and Engineering Innovation Research Group, School of Anti-Aging and Regenerative Medicine, Mae Fah Luang University, Bangkok 10110

Thailand.

Orcid ID:

Dr. Phakkharawat Sittiprapaporn-D https://orcid.org/0000-0002-4103-9396

Source of Support: Brain Science and Engineering Innovation Research Group, Mae Fah Luang University (MFU-grant), Conflict of Interest: None. 